\title{
A Review of Active Magnetic Bearings Supported Systems Optimization Design
}

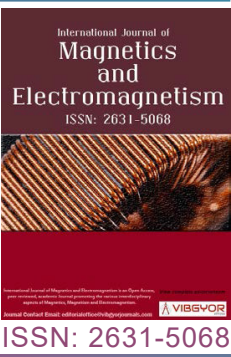

\section{Wan Zhong*}

Solar Turbines, USA

\begin{abstract}
Active magnetic bearings (AMBs) have attracted a lot of attention due to their various advantages from its noncontact and actively controlled features. The challenges of the further wide spread of $A M B s$ include the extensive knowledge required in designing the complex system and thus the high design cost. Multi-objective Optimization solves this challenge by providing $A M B$ designers multiple solutions to achieve the best compromised objectives. Different optimization techniques and their applications onto AMBs are summarized. Future trends of AMBs supported system optimization are also discussed.
\end{abstract}

\section{Keywords}

Active magnetic bearings, Multi-objective optimization, Genetic algorithms

\section{Introduction}

An active magnetic bearing (AMB) system, which supports or levitates a shaft in a magnetic field controlled by position feedback, has attracted a lot of attention due to its special features and various advantages over conventional bearings. Its non-contact nature makes magnetic bearings have minimal friction and wear, which allows magnetic bearings to have higher efficiency and higher running speeds of the rotor. No lubrication is required in the $A M B$ system, therefore there are no oil contamination problems [1]. As a result, magnetic bearings can be used in harsh environments, including vacuum, cryogenic, gravity free, subsea, and high temperature environments. Without contact or wear on system components and oil contamination issues, the maintenance is reduced substantially. Magnetic bearings are actively controlled and have high static stiffness, which can provide precise control of the nominal shaft center.

Due to these advantages, the applications of magnetic bearings increase dramatically in a wide range of industrial machines, for example, motors, pumps, generators, compressors, blowers, and turbines. They have also been applied on energy storage flywheels, maglev trains, and Medicare devices like artificial hearts and heart pumps.

However, magnetic bearings have their limits and challenges. Magnetic bearings load capacity and specific load are limited by the saturation in magnetic material. The best magnetic conducting medium (iron cobalt alloy) has a saturation flux intensity of about 2.3 Tesla. Compared with conventional oil bearings which are passive and absorb energy, magnetic bearing supported system is active and can excite any mode within the bandwidth of

*Corresponding author: Wan Zhong, Solar Turbines, San Diego, CA, 92123, USA

Accepted: November 03, 2020; Published: November 05, 2020

Copyright: @ 2020 Zhong $\mathrm{W}$, et al. This is an open-access article distributed under the terms of the Creative Commons Attribution License, which permits unrestricted use, distribution, and reproduction in any medium, provided the original author and source are credited.

Zhong. Int J Magnetics Electromagnetism 2020, 6:027

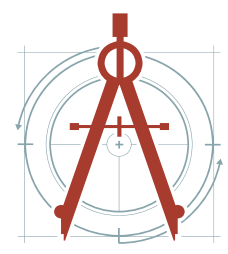


the controller. As a result, control of many modes, even beyond the operating speed range, is required for active magnetic bearings supported systems [1]. Time, cost, and advanced knowledge of both mechanical engineering and electrical engineering are required for the magnetic bearing/ rotor and control system design. Many of these challenges of magnetic bearings are in the design stage. As a result, optimization design, which aims to design a compact, highly efficient, reliable, and even auto tuned magnetic bearing system, is significant and has become a trendy topic in the area of AMBs.

The rest of this paper reviews different optimization algorithms, basic working principle of AMBs, different types of AMBs, current research status and future trend of the optimization design of AMBs.

\section{Optimization Algorithms}

\section{Multiple-objective optimization}

Multiple-objective optimization, which optimizes more than one goal simultaneously, have been widely used in various engineering applications, especially where optimal decisions need to be taken in the presence of trade-offs between two or more conflicting objectives. A mathematical definition of multiple objective optimization problem can be addressed as:
Minimize (or maximize)

$f_{i}\left(x_{1}, x_{2}, \ldots, x_{n}\right), i=1,2 \ldots p$

Such that

$h_{j}\left(x_{1}, x_{2}, \ldots, x_{n}\right)<0, j=1,2, \ldots q$

Where $\left(x_{1}, x_{2}, \ldots, x_{n}\right)$ are the $n$ design variables, $f_{i}\left(x_{1}, x_{2}, \ldots, x_{n}\right)$ are the $p$ objective functions, $h_{j}\left(x_{1}, x_{2^{\prime}}\right.$ $\left.\ldots, x_{n}\right)$ are the $q$ inequality constraints.

Pareto optimal solutions: In the single-objective optimization problem, the superiority of a solution over other solutions can be easily determined by comparing their objective function values. For multiple objective optimization problems, due to the conflict among the goals, there does not necessarily exist a single unique set of solutions that is the best for all objective functions. There usually are multiple sets of solutions, that none of the objectives can be improved without sacrificing any other objectives. Such solutions are called Pareto optimal solutions. Figure 1 shows an example of the Pareto optimal points in a two-objective optimization problem. These points form a Pareto frontier, on which objective 1 can only be minimized with the sacrifice of a larger objective 2 value and vice versa.

Objective evaluation: For multiple-objective optimization, many classical optimization algorithms simply use weight factors to combine all the objectives into one, which eventually turns the problem

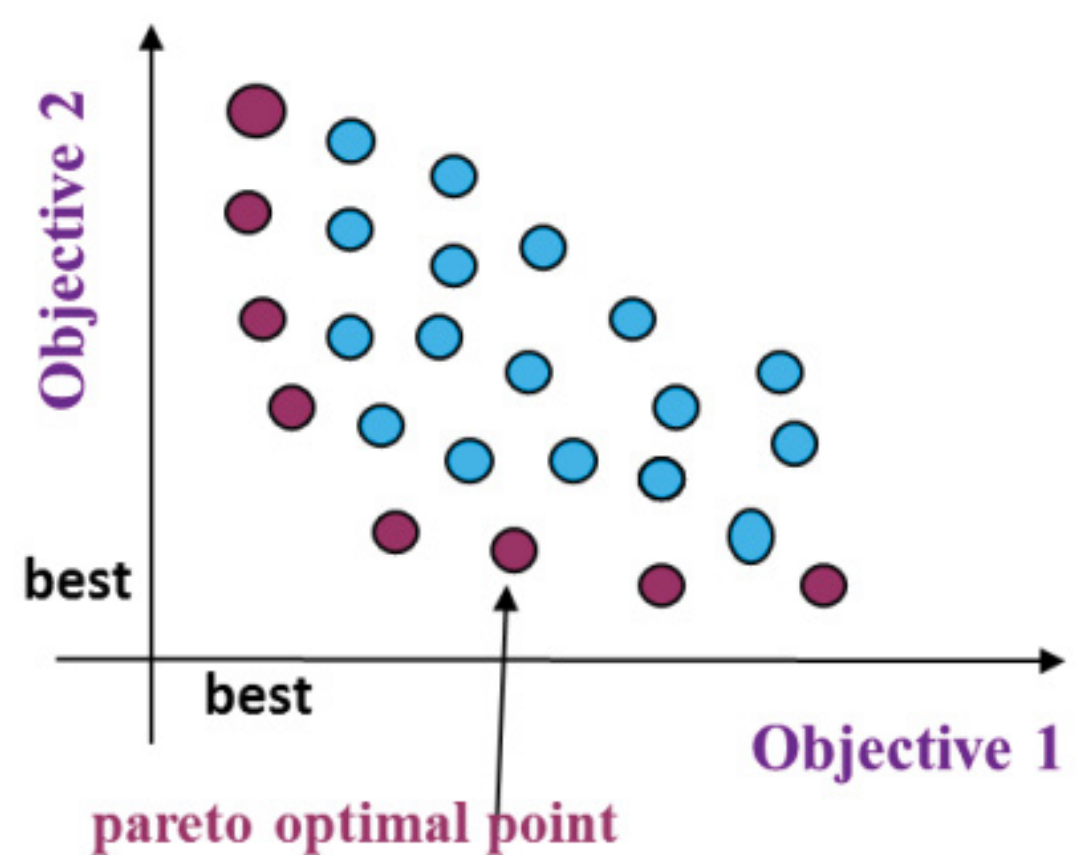

Figure 1: Pareto's frontier and Pareto solutions in optimization with two objectives. 
into single-objective optimization and searches for the single unique solution. However, in many real applications, it is difficult to define such weight factors to obtain a solution in a desired region in the objective space.

Constraint handling: Traditional optimizations use penalty function method to convert constrained problems into unconstrained problems by introducing penalty parameters for violating the constraints. Usually, a penalty function approach uses a penalty parameter proportional to the extent of constraint violation. If the penalty parameter is too small, the constraints are not emphasized enough, thereby leading to infeasible solutions. If a penalty parameter is too large, the problem will behave like a constraint satisfaction problem, thereby leading the problem to find an arbitrary feasible solution without enough emphasizing on the objectives.

In the next session, we will introduce several different multiple-objective optimization techniques and how they evaluate objective functions and handle constraints.

\section{Multiple-objective optimization techniques}

Various multi-objective optimization techniques have been developed by previous researchers. Traditional multi-objective optimization methods such as Weighted Sum Method, $\varepsilon$-Constraint Method [Haimes, et al. 1971], and Weighted Metric Method. Weighted Sum Method scalarizes a set of objectives into a single objective by adding each objective by a user defined weight factor, which is simple to use. However, in real applications it is hard to set these weight factors. $\varepsilon$-Constraint Method keeps only one of the objectives and restricts the rest within user-specific values $\varepsilon$. The $\varepsilon$ vector has to be chosen carefully so that it is within the minimum or maximum values of the individual objective function. The Weighted Metric Method combines multiple objectives using the weighted distance metric of any solution from the ideal solution, which requires knowledge of minimum and maximum objective values. All these three traditional multi-objective optimization techniques convert the multi-objective into one single objective function only, and require users' knowledge of weight factors, or minimum and maximum values of the objective functions.

Genetic Algorithms (GAs) are adaptive heuristic search algorithms based on the evolutionary ideas of natural selection and genetics [2]. Based on the number of optimization objectives, there are Single Objective Genetic Algorithms (SOGAs) and Multi-Objective Genetic Algorithms (MOGAs). In GAs, the evolution starts from a population of randomly generated individuals which is called initial generation. The fitness of each individual, which is usually the objective function value, is evaluated in each generation. The individuals with better objective functions are selected from the current population as parents to crossover and mutate to reproduce offspring as a new generation. The new generation is then used in the next algorithm iteration. The iteration terminates when either a maximum number of generations has been produced of a satisfactory level of fitness is reached for the population. Figure 2 is the flow chart of the GAs.

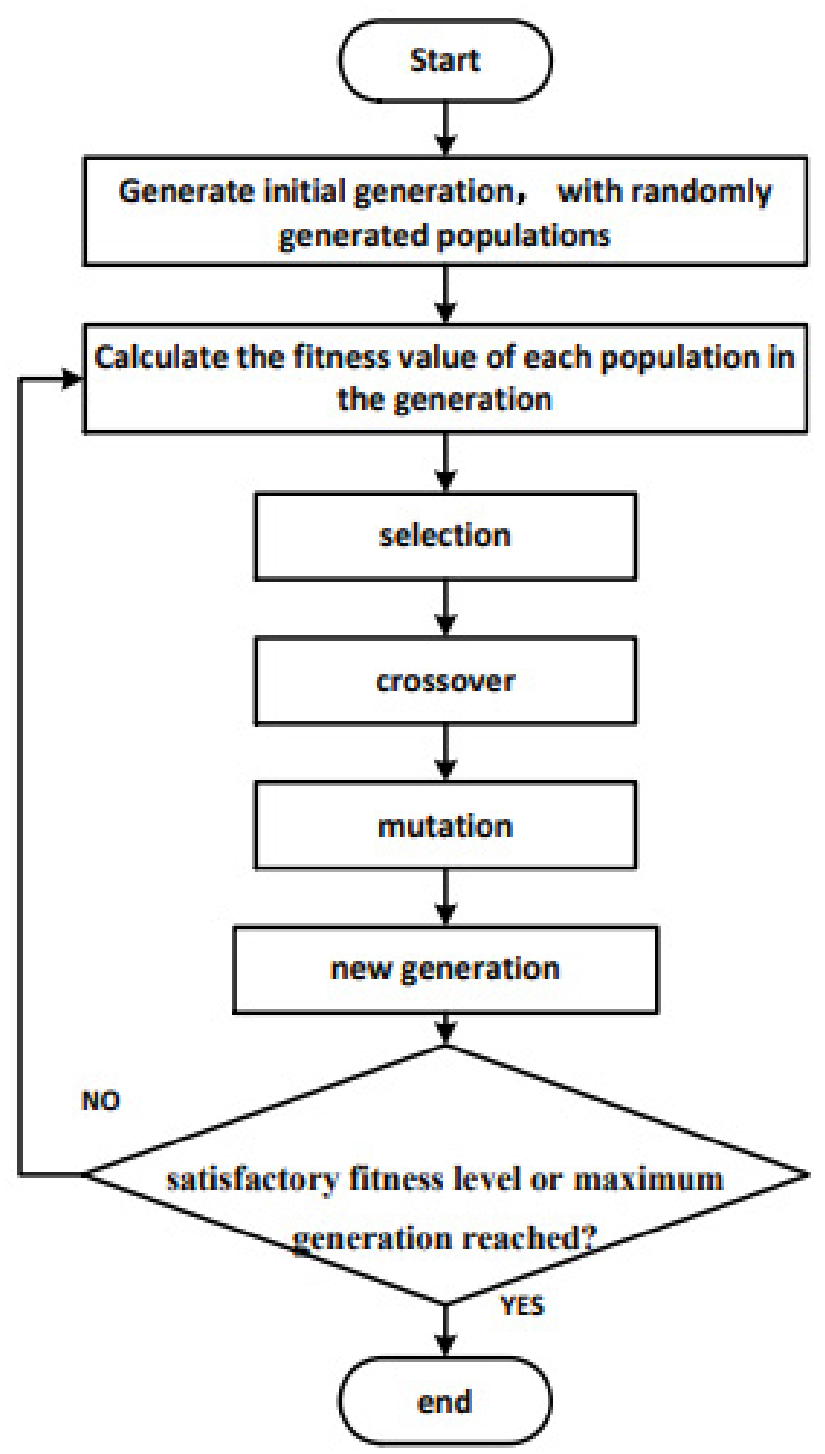

Figure 2: Flowchart of genetic algorithms. 
GAs have become one of the most popular optimization techniques, especially in solving multi-objective problems due to a lot of advantages over traditional methods. GAs work with a large number of variables that many parameters can be optimized, and they search from a wide sampling of the cost surfaces simultaneously to provide a list of optimum solutions rather than just a single solution.

The Non-dominated Sorting Genetic Algorithm-II (NSGA-II) optimization techniques by Deb, et al. [3] have been applied to many different design problems and work as a standard that other algorithms are compared with. NSGA-II uses the same selection, crossover, and mutation operations as the standard genetic algorithms but sorts the populations by "nondominated sorting" and "crowding distance sorting" before the selection. It uses the binary tournament selection for constraint handling, in which all the solutions are ranked by feasibility and the constraint violation. Feasible solutions have a better nondomination rank than the infeasible solutions. All the feasible solutions are ranked based on the objective function values. All the infeasible solutions are ranked in the way that a solution with a smaller constraint violation has a better nondomination rank. This is a non-penalty constraint handling technique and there will be penalty factors related issues.

Neighborhood Cultivation Genetic Algorithms (NCGA) by Watanabe, et al. [4], was developed from NSGA-II and includes the mechanisms of NSGA-II with standard genetic operation of mutation and crossover. On top of these standard operations, NCGA also uses the mechanism of neighborhood crossover, in which the parents are selected from populations with values close to one of the objectives while in normal crossover the parents are chosen randomly from the populations. NCGA has been demonstrated to have better solutions than NSGA-II and widely used.

Strength Pareto Evolutionary Algorithm (SPEA2) [5] by Zitzler, et al. also received a lot of attention because of their simplicity and effectiveness.

Table 1: Multiple objective optimization algorithms comparison.

\begin{tabular}{|c|c|c|}
\hline Optimization Method & Advantages & Disadvantages \\
\hline Weighted Sum Method & - Simple & $\begin{array}{l}\text { - Difficult to set the weight factors } \\
\text { - Not applicable to non-convex } \\
\text { problems }\end{array}$ \\
\hline$\varepsilon$-Constraint Method & $\begin{array}{l}\text { - Applicable to both convex and non- } \\
\text { convex problems }\end{array}$ & $\begin{array}{l}\text { - The } \varepsilon \text { vector has to be chosen } \\
\text { carefully }\end{array}$ \\
\hline Weighted Metric Method & $\begin{array}{l}\text { - Guarantees finding all Pareto-optimal } \\
\text { solutions with ideal solution }\end{array}$ & $\begin{array}{l}\text { - Requires min and max objective } \\
\text { values } \\
\text { - Requires that ideal solution can be } \\
\text { found by independently optimizing } \\
\text { each objective function }\end{array}$ \\
\hline NSGA-II \& NCGA & $\begin{array}{l}\text { - Non-penalty constraint handling } \\
\text { - Fast and efficient convergence } \\
\text { - Search in a wide range and handle } \\
\text { problems that start with non-feasible } \\
\text { solutions }\end{array}$ & - Complexity \\
\hline SPEA2 & $\begin{array}{l}\text { - Once a solution on Pareto-optimal } \\
\text { front is found, it is stored in the } \\
\text { external population } \\
\text { - Advantages from NSGA-II }\end{array}$ & $\begin{array}{l}\text { - Requires a balance between the } \\
\text { regular population size and the } \\
\text { external population size }\end{array}$ \\
\hline MOPSO & - Simple & $\begin{array}{l}\text { - Worse than GAs in finding pareto } \\
\text { solutions }\end{array}$ \\
\hline MOSA & - $\quad$ Fast & $\begin{array}{l}\text { - Worse than GAs in finding pareto } \\
\text { solutions }\end{array}$ \\
\hline
\end{tabular}


In SPEA2, the quality or fitness of an individual is measured considering its Pareto strength and its distance to its neighbor. It uses external populations to archive fixed number of non-dominated solutions. Newly found non-dominated solutions are compared with the existing external population and the resulting non-dominated solutions are preserved. SPEA2 uses nearest neighbor density approach to maintain the diversity and archive truncation method to preserve the boundary solutions. SPEA2 uses the same mutation and crossover operators of NSGA-II. It has been widely used in different multiple objective optimization problems and compared with NSGA-II [6-8].

Other multiple objective algorithms have also been developed, for example Multi-objective Particle Swarm Optimization (MOPSO) by Coello, et al. [9] and Multi-objective Simulated Annealing (MOSA) [10]. However, there are no significant advantages of the MOPSO over genetic algorithms, other than the simplicity of programming. MOSA is faster than genetic algorithms in speed. But if given enough time, genetic algorithms demonstrate better Pareto solutions than MOSA. Table 1 summarizes the advantages and disadvantages of these optimization algorithms.

\section{Optimization of AMB Supported Systems}

The main components in a closed loop controlled AMB system include a rotor, sensors, controllers, power amplifiers, and magnetic bearing actuators. Figure 3 depicts a simplified closed loop of the system. A sensor measures the displacement of the rotor from its reference position; a controller

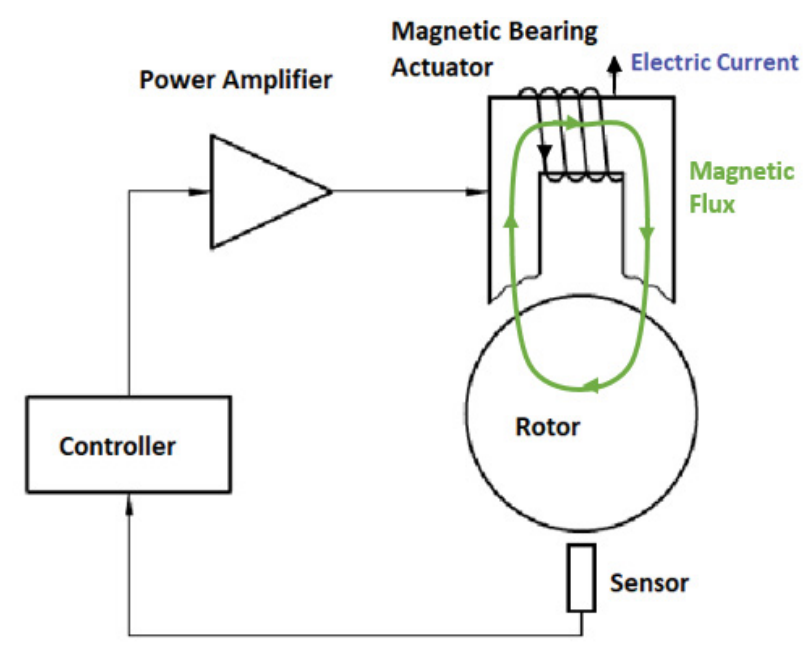

Figure 3: Simplified closed loop of magnetic bearings supported systems. converts the sensor signal into a control signal; a power amplifier transforms this control signal into a control current; and the control current generates magnetic forces in the magnetic bearing actuator to suspend the rotor to the desired position.

Depending on the direction of forces that AMBs support a rotor, there are radial/journal $A M B s$ and axial/thrust AMBs. Usually, a radial AMB levitates a rotor against gravity and/or supports it against unbalance forces. An axial/thrust AMB usually supports a rotor against axial forces like unbalance force from fluid. It can also levitate a vertical rotor against gravity. A lot of studies have been done to optimize different types of AMB actuators.

For axial AMBs, Spece, et al. [11] optimized the axial AMB actuators by generating Pareto-optimal curves to explore the influence that disk outer radius, peak force, axial gap, and magnetic permeability have upon actuator bandwidth. This actuator geometry optimization for bandwidth was carried out via MATLAB's fmincon function, which uses an interior-point algorithm to find the set of decision variables that maximize the objective functions, while satisfying the geometric constraints. This fmincon function is essentially a single objective nonlinear optimization. Besides, this optimization technique does not guarantee a global maximum. The authors had to repeat with various starting points to verify that the maximum found was unique and represented a global maximum. Rao, et al. used multiple objective genetic algorithms to optimize thrust bearings, with the power loss and the actuator weight selected as two optimization objectives [12]. They further optimized double-acting hybrid magnetic thrust bearings using multiple objective evolutionary algorithms [13]. Five objectives, including Minimization of the power loss, the weight, the control input and dynamic performance indices and maximization of the load capacity are optimized simultaneously. Double-acting actuators and PD controllers are optimized together as one system. The authors also studied the convergence by varying the population size and the number of generations. In both optimization studies by Rao, et al., they included the saturation of magnetic flux density and the saturation of coil current density as constraints in the model and observed the performance with these two saturations. Zhang, et al. [14] proposed a new type of no-thrust-disk/thrust hybrid magnetic bearing designed to reduce wind 
loss in centrifugal compressors. An integrated optimization method, which combines Genetic Algorithms (GA) with sequential quadratic programming (SQP) was used to optimize the actuator mass and its axial length. This study incorporated the flux leakage and eddy current losses into the optimization model and the losses were validated with 3D FEM analysis. However, the optimization still used weight factors to sum all the objectives into one function. The penalty functions are also used in the optimization.

Radial magnetic bearings are generally classified into heteropolar magnetic bearings (HEMB) as shown in Figure 4 and homopolar magnetic bearings (HOMB) as shown in Figure 5. For the HEMBs, the magnetic pole polarities alternate in the rotational plane, while all the poles of HOMBs have the same polarity in the rotational plane. This same polarity in the rotational plane reduces the eddy current losses in the HOMBs and they are thus more efficient than HEMBs. As a result, HEMBs are more commonly used for their simple structure and low cost, while HOMBs are usually used in applications where compact design and high efficiency are preferred over cost, for example aerospace.

A lot of research has been done to optimize the HEMB designs due to their wide applications. Lijesh, et al. [15] optimized the magnetic levitation force for an eight pole HEMB radial bearing, with constraints from geometric considerations and power losses. This single objective optimization nonlinear constraint problem was solved with Matlab Toolbox. Carlson-Skalak and Maslen, et al. [16] used single objective genetic algorithms (SOGAs) to optimize the HEMB actuators. The concept of catalog selection was used, which made the optimization more amenable to commercial design. Chang, et al. [17] used SOGAs to minimize the actuator volume of the HEMB with given static load, dynamic load and the equivalent stiffness. The sta-

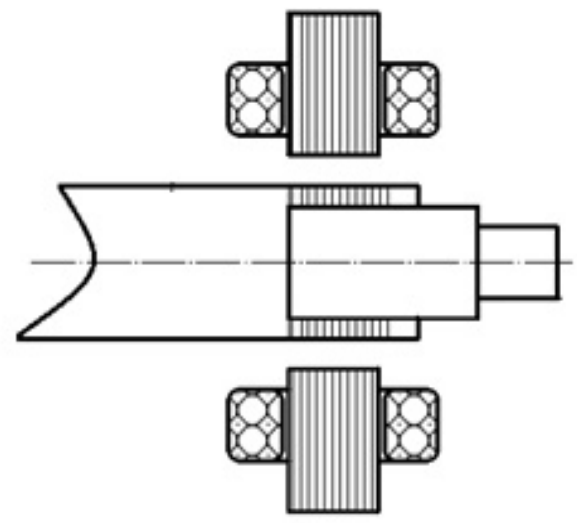

(a)

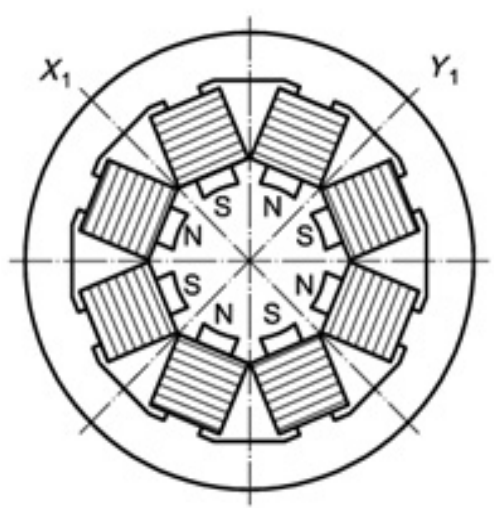

(b)

Figure 4: Heteropolar radial magnetic bearing with alternating poles.

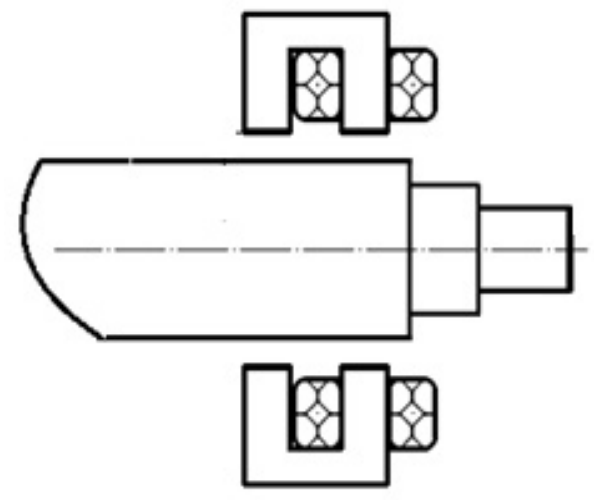

(a)

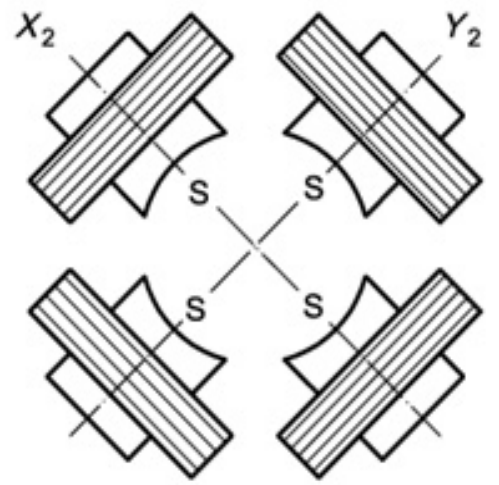

(b)

Figure 5: Homopolar radial magnetic bearing actuator with same poles. 
bility of the closed loop system, including the controller and power amplifier was included. Reddy, et al. [18] optimized a HEMB actuator AMB for a compact size using SOGAs. Three different SOGAs techniques, adaptive multipopulation genetic algorithm (AMPGA), multipopulation genetic algorithm (MPGA) and simple genetic algorithm (SGA) were compared. AMPGA was demonstrated better in optimizing the objective function.

Shelke, et al. [19] used multi-objective genetic algorithms (MOGAs) to optimize both the actuator weight and the copper losses in the coil of the HEMB actuator. The NSGA-II was selected as an optimization tool, coil dimensions and number of poles were used as design variables. Ren, et al. [20] optimized the power loss and mass of HEMB for a vacuum turbo-molecular pump using MOGAs. Experiments on a prototype pump were carried out to verify the optimization design. Le, et al. [21] combined GAs and branch and bound (BNB) algorithm to optimize the design of an eight pole $\mathrm{HEMB}$ supported rotor-bearing system. The design objectives in this study include maximizing the static load capacity, minimizing the volume and the mass, or minimize the power loss. GAs can find solutions in the broader search space and get the global optimum solution, while BNB is better in finding the optimal solution in the local search. A combination of these two algorithms is demonstrated to be better in optimizing objectives. As can be seen, the optimization of HEMBs are focused on designing the actuator to improve reduce weight, pow losses, or to improve dynamic performance. GAs and modified GAs have been the most popular algorithms in multiple objective optimizations of magnetic bearings supported systems.

Although $\mathrm{HOMBs}$ are more compact and energy efficient designs, the research on HOMBs, especially the optimization design of HOMBs has not been as popular as HEMBs. Cao, et al. [22] used Generalized Self-Adapting Particle Swarm Optimization (GAPSO) to optimize a HOMB actuator. The actuator volume and the core losses on the actuator are set as two objectives and are optimized simultaneously. The results show improved thermal performance with reduced power loss and temperature rise. However, in the power loss analysis, only copper losses and core losses were included, while windage losses which could be dominate at high speed were ignored. Zhong, et al. [23] used NSGA-II to optimize the HOMB actuator and its PD controller in a closed loop-controlled system. The HOMB actuator mass, the steady state vibration amplitude from transient analysis, and the power loss including copper losses, core losses and windage losses are set as three minimization type objectives.

Combo magnetic bearings are those combine radial and axial bearing in one unit. This has been a popular design in industry. However, not so much optimization has been done on this type of magnetic bearings. Han, et al. [24] optimized a combined radial-axial magnetic bearing for a magnetically suspended compressor using multiple objective optimizations. Total volume, axial length, and radial force were selected as three objectives. This multiple objective optimization used weighting factors to combine five different objectives into one objective function. This combined objective function is then optimized with Evolutionary Algorithms and sequential quadratic programming (SQP). The optimization results were verified by Finite Element Method analysis and prototype experiments.

The control strategies of the AMB system are also optimized. Schroder, et al. [25] used the genetic algorithms to optimize the robust control strategy of the 5-axis controlled HEMBs supported rotordynamic system with both radial bearing and axial bearing. Chen [26] proposed a multi-objective optimization method for the parameter tuning of conventional PID controllers of HEMBs supported systems based on an improved adaptive genetic algorithm (AGA). He later used the same AGA to tune the parameter of fuzzy PID and fractional PID controllers $[27,28]$. The conventional PID controllers have three constants, proportional constant, integral constant, and derivative constant. The fuzzy PID combines conventional PID controllers with fuzzy logic. The fractional PID controllers have two more constants for derivative order and integral order. They both are more flexible in adjusting the closed-loop system characteristics than conventional PID controllers. The studies show that both the optimized fuzzy PID controller and the fractional PID controller have good performance in both transient and steady state stages Jastrzębski, et al. [29] used genetic algorithms to optimize the controller parameters of a closed-loop centralized optimal position control strategy for a multi-input multi-output HEMBs system. The system performance is compared with the one controlled by the 
classical PID controller and demonstrated to have better performance. Hsiao, et al. [30] used the goal programming as the search method and finite difference to optimize the HEMB system controlled with a PD controller. The optimization objectives are to maximize the force slew rate and minimize the displacement sensitivity at large displacements. In this linearized model, the maximum flux intensity, maximum supply voltage, are added to constraints to limit the flux and voltage saturation. The optimization shows that the pole width, coil turns, and pole length are critical design variables affecting the force slew rate while the proportional gain of the PD controller dominates the displacement sensitivity. Zhong, et al. [31] used NSGA-II and NCGA to optimize an eight-pole HEMB actuator and PD controller simultaneously. Three objectives were selected, including minimization of the actuator mass, minimization of the power loss, and maximization of the external static load capacity in a transient analysis of the rotor. Zhong, et al. [23] also used NSGA-II to optimize a HOMB actuator and its controller, with a two-stage control approach utilized, which used different PD controllers for the rotor levitation/startup, and at a steady state operating speed with unbalance. Modern controls like active disturbance rejection control [32,33], robust control [34-36], sliding mode control [37-39], repetitive control $[40,41]$, neural network control [42] have been applied into magnetic bearing supported systems. However, when it comes to optimization design of the magnetic system, most researchers still use conventional PID controller.

\section{Future Research}

Most of the current researches on magnetic bearings are still focused on the heteropolar radial magnetic bearings due to their simple geometry and wide applications. However, the homopolar radial magnetic bearings, which usually use permanent magnets to provide bias flux, are more compact in size and efficient in size. The more widely spread of homopolar magnetic bearings are limited by its complex geometry and therefore extra design efforts. Optimization design has the great potential to solve the design challenges and reduce design cost of HOMBs and thus can facilitate the widespread use of such state-of-the-art and efficient AMB.

Although many current researchers have integrated the controller design into the optimization of the AMBs supported system, most of them use conventional or fractional PID/PD controllers. As the development of AMB technology, the PID/PD controller alone has been unable to meet the growing demands. New modern control strategies, such as fuzzy control, neural network and other intelligent control methods are required to improve the control performance. The optimization design with such modern control strategies integrated is also in demand to meet the needs from industrial applications.

Most of the current magnetic bearings research as well as the optimization design limits the system in the linear range, which means that flux saturation or current saturation are not allowed. The maximum flux and current are defined and used as optimization constraints. However, some extend of flux saturation could help improve the system load capacity. Besides, the linear model of the magnetic bearing systems, which linearizes the electromagnetic force with respect to the control current and rotor displacement and ignores the nonlinearities from system components including power amplifiers, sensors, and actuators, could be misleading. A high-fidelity nonlinear model which allows the system into transient or temporary saturation along a section of the flux path could help improve the system performance.

\section{Conclusions}

In this paper, the advantages and limits of active magnetic bearings supported systems are introduced. Different types of optimization techniques along with their advantages and disadvantages are compared. Current research status of the optimization design on magnetic bearings and future trends are discussed.

\section{References}

1. (2004) Mechanical vibration-Vibrations of rotating machinery equipped with active magnetic bearings-Part, 4. Standard I, 14839-4.

2. Sivaraj R, Ravichandran T (2011) A review of selection methods in genetic algorithm. International Journal of Engineering Science and Technology (IJEST) 3: 3792-3797.

3. Deb K, Pratap A, Agarwal S, Meyarivan T (2002) A fast and elitist multiobjective genetic algorithm: NSGA-II. Evolutionary Computation, IEEE Transactions 6: 182-197. 
4. Watanabe S, Hiroyasu T, Miki M (2002) NCGA: Neighborhood Cultivation Genetic Algorithm for multi-objective optimization problems. Proc. GECCO Late Breaking Papers, 458-465.

5. Zitzler E, Laumanns M, Thiele L (2001) SPEA2: Improving the strength pareto evolutionary algorithm. Eidgenössische Technische Hochschule Zürich (ETH), Institut für Technische Informatik und Kommunikationsnetze (TIK).

6. Tomoyuki H, Nakayama S, Miki M (2005) Comparison study of SPEA2+, SPEA2, and NSGA-II in diesel engine emissions and fuel economy problem. 2005 IEEE congress on evolutionary computation, 1.

7. King RTF Ah, Deb K, Rughooputh HCS (2010) Comparison of NSGA-II and SPEA2 on the multiobjective environmental/economic dispatch problem. University of Mauritius Research Journal 16: 485-511.

8. Bhargav G, Savsani V, Patel V (2016) Multi-objective optimization of vehicle passive suspension system using NSGA-II, SPEA2 and PESA-II. Procedia Technology 23: 361-368.

9. Coello Coello CA, Lechuga MS (2002) MOPSO: A proposal for multiple objective particle swarm optimization. Proceedings of the 2002 Congress, IEEE, 10511056.

10.Nam D, Park CH (2000) Multiobjective simulated annealing: A comparative study to evolutionary algorithms. International Journal of Fuzzy Systems 2: 87-97.

11.Spece H, Fittro R, Knospe C (2018) Optimization of axial magnetic bearing actuators for dynamic performance. Actuators 7: 66.

12. Rao JS, Tiwari R (2008) Optimum design and analysis of thrust magnetic bearings using multi objective genetic algorithms. International Journal for Computational Methods in Engineering Science and Mechanics 9: 223-245.

13.Rao JS, Tiwari R (2009) Design optimization of double-acting hybrid magnetic thrust bearings with control integration using multi-objective evolutionary algorithms. Mechatronics 19: 945-964.

14.Zhang P, Wang Z, Xi G (2019) A multi-objective optimal design methodology for solid core no-thrustdisk/thrust hybrid magnetic bearings considering eddy-current effects and leakage. International Journal of Applied Electromagnetics and Mechanics 61: 13-42.

15.Lijesh KP, Hirani H (2015) Optimization of eight pole radial active magnetic bearing. Journal of Tribology, 137.
16.Carlson-Skalak S, Maslen E, Teng Y (1999) Magnetic bearing actuator design using genetic algorithms. Journal of Engineering Design 10: 143-164.

17.Chang H, Chung SC (2002) Integrated design of radial active magnetic bearing systems using genetic algorithms. Mechatronics 12: 19-36.

18. Reddy AS, Agarwal PK, Chand S (2019) An adaptive multipopulation genetic algorithm for the optimization of active magnetic bearings. IOP Conference Series, Materials Science and Engineering 691: 012009.

19. Shelke S, Chalam R (2011) Optimum power loss analysis of radial magnetic bearing using multi-objective genetic algorithm. International Journal of Computer Applications 27: 20-27.

20.Ren X, Le Y, Han B, Wang K (2017) Loss optimization and thermal analysis of a heteropolar magnetic bearing for a vacuum turbo-molecular pump. International Journal of Applied Electromagnetics and Mechanics 54: 673-690.

21.Le Y, Fang J, Wang K (2014) Design and optimization of a radial magnetic bearing for high-speed motor with flexible rotor. IEEE Transactions on Magnetics 51: 1-13.

22.Cao Y, Liu C, Zhu S, Yu J (2019) Temperature field analysis and optimization of the homopolar magnetic bearing. Progress In Electromagnetics Research 85: 105-114.

23.Zhong W, Palazzolo A (2015) Magnetic bearing rotordynamic system optimization using multi-objective genetic algorithms. Journal of Dynamic Systems, Measurement, and Control 137: 021012.

24. Han B, Xu Q, Yuan Q (2015) Multiobjective optimization of a combined radial-axial magnetic bearing for magnetically suspended compressor. IEEE Transactions on Industrial Electronics 63: 2284-2293.

25.Schroder P, Green B, Grum N, Fleming P (2001) Online evolution of robust control systems: An industrial active magnetic bearing application. Control Engineering Practice 9: 37-49.

26.Chen HC (2008) Adaptive genetic algorithm based optimal PID controller design of an active magnetic bearing system. Innovative Computing Information and Control, 3rd International Conference, IEEE, 603-603.

27. Chen HC (2008) Optimal fuzzy pid controller design of an active magnetic bearing system based on adaptive genetic algorithms. Machine Learning and Cybernetics, 2008 International Conference, IEEE, 2054-2060. 
28.Chang LY, Chen HC (2009) Tuning of fractional PID controllers using adaptive genetic algorithm for active magnetic bearing system. WSEAS Transactions on systems 8: 158-167.

29.Jastrzębski RP, Pöllänen R (2009) Centralized optimal position control for active magnetic bearings: comparison with decentralized control. Electrical Engineering 91: 101-114.

30.Hsiao FZ, Fan CC, Chieng WH, Lee AC (1996) Optimum magnetic bearing design considering performance limitations. Jsme Int J C-Dyn Con 39: 586-596.

31.Zhong W, Palazzolo A, Kang X (2017) Multi-objective optimization design of nonlinear magnetic bearing rotordynamic system. Journal of Vibration and Acoustics, 139.

32.Alexander BXS, Rarick R, Dong L (2007) An application of active disturbance rejection control to self-sensing magnetic bearings. 2007 American Control Conference, IEEE.

33.Wang S, Zhu H, Wu M, Zhang W (2020) Active disturbance rejection decoupling control for three-degreeof- freedom six-pole active magnetic bearing based on bp neural network. IEEE Transactions on Applied Superconductivity 30: 1-5.

34.Erkki L (1999) Robust control of magnetic bearings in subcritical machines. Helsinki University of Technology.

35.Carl RK, Tamer SM (1997) Experiments in robust control of rotor unbalance response using magnetic bearings. Mechatronics 7: 217-229.
36.Zdzisław G, Mystkowski A (2008) Robust control of active magnetic suspension: analytical and experimental results. Mechanical Systems and Signal Processing 22: 1297-1303.

37.Alok S, Mease KL, Wang KW (1991) Sliding mode control of a rigid rotor via magnetic bearings. 1991 ASME Design Technical Conference presented at the 13th Biennial Conference on Mechanical Vibration and Noise, - Miami, FL, USA.

38.Jun-Ho L, Allaire PE, Tao G, Decker JA, Zhang X (2003) Experimental study of sliding mode control for a benchmark magnetic bearing system and artificial heart pump suspension. IEEE Transactions on control systems technology 11: 128-138.

39.Zad HS, Irfan Khan T, Lazoglu I (2017) Design and adaptive sliding-mode control of hybrid magnetic bearings. IEEE Transactions on Industrial Electronics 65: 2537-2547.

40.Tsu-Chin T, Qian YX, Nemani M (2000) Repetitive control for asymptotic tracking of periodic signals with an unknown period. J Dyn Sys Meas Control 122: 364-369.

41.Zhang X, Shinshi T, Li L, Shimokohbe A (2003) A combined repetitive control for precision rotation of magnetic bearing. Precision engineering 27: 273282.

42. Choi H, Buckner G, Gibson N (2006) Neural robust control of a high-speed flexible rotor supported on active magnetic bearings. 2006 American Control Conference, IEEE. 